\title{
VARIACIÓN INTRADIALECTAL DEL ESPAÑOL: IDEOLOGÍAS LINGÜÍSTICAS EN LA JUVENTUD DE
}

\section{FUENLABRADA}

\author{
Daniel Pinto Pajares \\ Universidad de Vigo \\ danielpinto_92@hotmail.es
}

Resumen: Las ideologías panhispánicas fomentadas por las instituciones académicas construyen el marco de un español general cohesionado y homogéneo que garantiza el entendimiento entre diferentes comunidades lingüísticas, frente a las múltiples realizaciones cotidianas que, por su carácter espontáneo y alejado de la normativa estándar, se presentan como versiones deturpadas. Mediante cuestionarios anónimos, hemos realizado una investigación cuantitativa que toma como objeto de estudio el alumnado de Bachillerato de Fuenlabrada. Estudiamos las ideologías lingüísticas que presenta una muestra representativa en torno a la variación intradialectal del español. El español estándar es contrapuesto a las variedades de España e Hispanoamérica y las ideologías lingüísticas detectadas engloban prejuicios respecto a ciertas variedades y a sus hablantes. Entre otras cuestiones, se observa una vinculación entre características estéticas como propiedades consustanciales a la lengua y una representación del estándar como ideal de corrección. No obstante, las variedades concretas y acentos no estándares no alcanzan percepciones negativas, así como se desligan las diferentes variedades del español con las características psicológicas de sus hablantes. Palabras clave: prejuicio lingüístico, español estándar, dialecto, panhispanismo, corrección lingüística

\begin{abstract}
Panhispanic ideologies promoted by academic institutions present a framework of a cohesive and homogeneous general Spanish language that guarantees understanding between different linguistic communities. This stands in opposition to the multiple daily realizations whose spontaneity and non-normative nature is presented as deviant. By means of anonymous questionnaires, we have carried out a quantitative research with Baccalaureate students from Fuenlabrada as objects of study. We analysed the linguistic ideologies presented in a representative sample regarding the intradialectal variation of the Spanish language. Standard Spanish is contradictory to the varieties of Spanish in Spain and Spanish America and the detected linguistic ideologies include prejudices with respect to certain varieties and their speakers. Among other issues, there is a link between aesthetic features as consubstantial properties to the language and a representation of the standard as an ideal of correction. Nevertheless, the concrete varieties and non-standard accents do not reach negative perceptions, just as the different varieties of Spanish are separated with the psychological characteristics of their speakers.

Keywords: linguistic prejudice, standard Spanish, dialect, panhispanism, linguistic correction
\end{abstract}




\section{INTRODUCCIÓN}

Con unos 400 millones de hablantes repartidos en 23 países, la realidad sociopolítica del español se caracteriza por su naturaleza transnacional. La evolución histórica del español en las múltiples comunidades lingüísticas se puede observar actualmente en las diversas variedades lingüísticas que coexisten, interactúan y reciben elementos de las lenguas con las que están en contacto. Este funcionamiento propio de las lenguas naturales es, sin embargo, visto por parte de instituciones académicas y de muchos hablantes como una desviación de un ideal de lengua homogénea. El centro hegemónico de la hispanofonía ha radicado históricamente en España, por lo que la variación lingüística del español se juzga con la mirada puesta en organismos como la RAE, cuyos trabajos de índole prescriptiva y descriptiva son tomados como reglas para un uso idiomático correcto, de tal manera que se reduce el valor de aquellas formas lingüísticas que contradicen la norma.

En esta investigación, estudiamos las ideologías lingüísticas que presenta el alumnado de Bachillerato de Fuenlabrada respecto a la variación intradialectal del español. La metodología empleada ha consistido en cuestionarios repartidos a una muestra representativa, de manera que a lo largo de este trabajo exponemos y cuantificamos las ideologías lingüísticas detectadas. En tanto que municipio periférico de la Comunidad de Madrid, Fuenlabrada no ha recibido gran atención por parte de la sociolingüística en estudios sobre el español. Consideramos que la exploración del área de Madrid desde una perspectiva sociolingüística del español resulta crucial puesto que su condición de centro político y en el que radican las academias de la lengua española de mayor prestigio articulan una influencia que trasciende los límites regionales e incluso nacionales.

Por lo tanto, el primer objetivo de esta investigación es plantear una problemática escasamente estudiada en localidades del extrarradio de Madrid que, como ya se ha referido, representa un campo de trabajo altamente influyente en varios niveles territoriales. El segundo objetivo radica en exponer las opiniones que presenta una muestra representativa respecto a la variación intradialectal del español y que son fomentadas por algunas instituciones académicas del panhispanismo.

\section{ANTECEDENTES}

El auge de la industrialización experimentado en los mayores focos urbanos de España a partir de la década de 1960 situó a la Comunidad de Madrid como uno de los principales centros de atracción de inmigrantes nacionales, especialmente del 
área meridional. Este flujo migratorio se asentó en la periferia de la capital, donde paralelamente se ubicaron las nuevas fábricas, dando lugar a una convergencia lingüística entre diferentes variedades del español (García Mouton y Molina Martos, 2009). Numerosos autores han señalado que la vinculación de Madrid con la idea de capitalidad y de modelo normativo no ha animado a la sociolingüística ni a la dialectología a emprender proyectos basados en las características del habla de Madrid (Paredes, 2006; Molina, 2009). Más bien, se ha aprovechado su condición de foco receptor de inmigración para estudiar el contacto lingüístico (Sancho Pascual, 2015; Solís Casco, 2015; Peralta Céspedes, 2017).

A pesar de ello, se pueden citar de manera fragmentaria algunos trabajos interesados en las hablas madrileñas urbanas, no solo de las variedades capitalinas (Cestero, Molina y Paredes, 2006), sino también de las variedades de las nuevas urbes que empezaban a consolidarse al sur y al este de la Comunidad tras la explosión demográfica a finales del siglo XX (Martín Butragueño, 1993; Blanco, 2004). El objetivo de algunos de estos trabajos de corte sociolingüístico es dar cuenta de las normas lingüísticas empleadas por hablantes cultos, recogiendo para ello encuestas y grabaciones para analizar especialmente aspectos sintácticos y léxicos (Quilis, 1966; Torres Martínez, 1981).

A principios del siglo XXI, se llevaron a cabo proyectos etnolingüísticos para acercarse al conocimiento del habla de Madrid. En el marco del Atlas Linguistique Roman -AliR - (Contini y Tuaillon, 1996-2009) y del Atlas Linguarum Europae - ALE - (Alinei y Viereck, 1997), se realizaron dos campañas de cuestionarios sociodialectales y conversaciones de grupo entre 2001 y 2005 en diversas localizaciones de la Comunidad de Madrid. Los resultados avalaron que la frontera lingüística de las innovaciones meridionales sigue situándose en Madrid (Molina, 2006), destacaron el abandono de formas léxicas rurales por la presión de la lengua estándar promovida en los medios de comunicación (García Mouton, 2007) y una actitud lingüística generalizada en el medio rural que reconoce el habla femenina como más educada y correcta (García Mouton, 2011). Otras investigaciones vinculadas al Proyecto para el Estudio Sociolingüístico del Español de España y de América - PRESEEA - han tratado aspectos fonéticos (Gil Peña, 2004), léxicos (Paredes, 2005) y discursivos (Molina, 2005) del habla de Madrid.

Entre las localidades del área metropolitana de Madrid, Fuenlabrada no ha recibido suficiente atención por parte de la investigación sociolingüística. Otros municipios colindantes como Getafe, que cuenta con un perfil demográfico análogo, han dado lugar a estudios sociolingüísticos relevantes. En el nivel fónico, Martín Butragueño (2001) y Gil Peña (2004) destacan diferencias relevantes entre el cinturón periférico de Madrid y los distritos de la capital en fenómenos como la aspi- 
ración o la elisión, que, por influencia de la inmigración meridional y extranjera, son más frecuentes en el sur de la Comunidad. Respecto a las actitudes lingüísticas, Martín Butragueño (1993) investiga los patrones que condicionan los cambios lingüísticos en la población procedente de la mitad sur de España y residente en Getafe. Entre otros resultados, destacan el desprestigio de las variedades de origen de las personas encuestadas y la tradicional concepción de las hablas castellanoleonesa y madrileña como las más correctas de la hispanofonía.

Ante la carencia de trabajos específicos sobre las ideologías lingüísticas de la juventud de Fuenlabrada respecto a la variación intradialectal del español, la originalidad de esta investigación contribuye a cubrir un vacío académico cuyo interés se justifica a partir de dos factores. Por un lado, el tramo de edad de la población investigada se relaciona con un sector lingüísticamente innovador e ideológicamente relevante para el futuro inmediato de las concepciones que se negocien y se asienten en la sociedad con respecto a las variedades del español. Por otro lado, Madrid es uno de los principales centros emisores de ideologías relacionadas con la lengua española, pero tal y como se ha observado en trabajos precedentes, el sur de la Comunidad, como en este caso lo es Fuenlabrada, configura un paradigma que difiere en distintos niveles lingüísticos con las localidades del centro y del norte, de manera que su carácter periférico puede condicionar las asunciones en torno a la variedad intradialectal del español.

\section{MARCO TEÓRICO}

En las últimas décadas, la lingüística ha puesto su atención en las opiniones, valoraciones y actitudes que los hablantes vinculan con ciertas lenguas y variedades lingüísticas. El pionero del concepto de "ideología lingüística" fue Silverstein (1979), pero su conceptualización estructuralista ha sido reinterpretada por otros autores que toman un punto de vista social. Irvine lo define como «the cultural system of ideas about social and linguistic relationships, together with their loading of moral and politics interests» (1989: 255) y las teorizaciones más recientes añaden un enfoque crítico que resalta la capacidad de las ideologías lingüísticas para producir y reproducir relaciones de poder (Kroskrity, 2010). Así, en los actos comunicativos se manifiestan los intereses políticos y económicos de diferentes grupos sociales en su pugna por generar un sentido común que racionalice la naturaleza de las lenguas y las nociones vinculadas con los hablantes (Mertz, 2010).

Estos atributos e ideas preestablecidas juegan un rol determinante en las formas de socialización entre distintas comunidades lingüísticas y en el propio seno 
de estas. En el contexto del español, la globalización favorece el contacto entre variedades, que implica la elaboración de estrategias para reconocer o para desafiar la variación característica de la lengua española (Bernárdez, 2010).

La planificación lingüística del mundo hispanohablante emana de instituciones como la Real Academia Española y la Asociación de Academias de la Lengua Española, que configuran un modelo de comunidad lingüística internacional en la que el español actúa como lugar común para los hispanohablantes y resaltan su potencialidad económica (Del Valle, 2007a). Estos organismos marcan un punto de referencia normativa fundado en elementos gramaticales primordiales que constituirían la base general de la lengua frente a la pluralidad. El modelo tradicional fomentado por la RAE se resume en su anterior lema «Limpia, fija y da esplendor», mediante el que se promovía un ideal homogéneo del español. Las variedades seleccionadas para representar la lengua culta estándar recayeron en las hablas castellanas, y más concretamente, en la lengua de la corte en Madrid dado su carácter de capital política y administrativa (Lauria y López García, 2009). En este sentido, diversos autores han señalado que los grupos políticos y económicos poderosos son los únicos capaces de imponer su propia variedad lingüística como modelo de prestigio, de lo cual no se deriva que estos dialectos contengan patrones intrínsecamente superiores, más avanzados o más correctos que los de otras variedades lingüísticas (Saussure, 1916; Penny, 2000).

La RAE ha adoptado desde las últimas décadas una nueva perspectiva consistente en el reconocimiento de la multiplicidad de variedades lingüísticas del español, pero siempre bajo una unidad liderada por el centro hegemónico del panhispanismo. Este cambio de enfoque se reconoce en su lema actual "Unidad en la diversidad". Bajo esta etiqueta, salen a colación conceptos como el de la lengua común, que iguala los diferentes modelos del español, pero, a su vez, encubre una norma impuesta y entiende el fomento de las lenguas en contacto con el español como ataques ilegítimos a la vitalidad sociolingüística de este (Del Valle, 2007b). A pesar de esta nueva conceptualización panhispánica que trata de compartir el poder estandarizador con diferentes academias hispanoamericanas, la RAE sigue manifestando implícitamente que el motor de cambio lingüístico y, por ende, de desviación, corresponde con las hablas americanas (Lauria y López García, 2009).

Sin embargo, la ciencia lingüística ha demostrado que el cambio es consustancial a toda lengua natural, por lo que cualquier intento de cohesionar sus elementos gramaticales y fonéticos a lo largo del tiempo está condenado al fracaso. Moreno Cabrera (2011) establece las siguientes propiedades esenciales de las lenguas. En primer lugar, la producción de una lengua varía tanto para el hablante individual como para la comunidad lingüística en cuestión, ya que las múltiples situaciones comunicativas condicionan los registros adoptados y ciertos factores como la clase social 
y la localización geográfica determinan el habla. En segundo lugar, el tiempo es un factor de cambio, de manera que distintas generaciones pueden presentar alteraciones sustanciales en la estructura lingüística y la acumulación de cambios a lo largo del tiempo puede dar lugar a lenguas nuevas. En tercer lugar, todo individuo está capacitado para adquirir espontáneamente la lengua del grupo al que se exponga, sin necesidad de una instrucción consciente. Este último punto conlleva la aceptación de que la lengua coloquial no es una formación deturpada de la lengua estándar, sino más bien lo contrario: ante numerosas realizaciones de un sistema lingüístico abstracto, las normas académicas plasmadas en el estándar culto son incapaces de recoger tal diversidad. Por tanto, no se puede asumir la idea de que, en determinadas circunstancias, los hablantes descuidan la lengua con arreglo a las pautas estandarizadas puesto que estos patrones son abstracciones de los fenómenos lingüísticos de una o varias realizaciones concretas, pero no de todas ellas.

El problema surge cuando se toma la lengua escrita, eminentemente artificial, como modelo de corrección. La carencia de polimorfismo característica de la lengua escrita y la presentación de la lengua en la enseñanza reglada a través de los códigos institucionalizados, tales como los diccionarios o las gramáticas, presupone la asunción de la lengua como un ente regular y atemporal. Ante las formas orales que contravienen la norma académica, los hablantes buscan en los diccionarios y en las gramáticas posibles soluciones que corrijan las supuestas desviaciones (Amorós Negre, 2008). No obstante, en toda variedad lingüística natural se pueden recopilar los patrones gramaticales que las rigen, pero la discrepancia con respecto a la norma estándar no la desautoriza, puesto que el estándar fijado a partir de una convención y el carácter relativamente invariable de la escritura son elaboraciones posteriores a la lengua coloquial y espontánea.

El carácter transnacional de lo que comúnmente se conoce como «lengua española » supone el reconocimiento de infinidad de variedades lingüísticas concretas cuyas delimitaciones desatan a menudo discrepancias dentro del mundo académico. El español es una idea abstracta de un conjunto de variedades observables que difieren entre sí en pequeños elementos y que, al comparar dos variedades geográficamente lejanas, el grado de variación aumenta a pesar de la mutua inteligibilidad (Moreno Cabrera, 2011). La suposición de algunas de estas variaciones como más o menos prestigiosas y correctas está motivada par razones extralingüísticas, puesto que ciertos criterios como el etimológico, entre otros, no anulan la legitimidad de otras formas lingüísticas. Es difícil caracterizar en bloque un español de España frente a un español de América, ya que el continuo dialectal conoce múltiples isoglosas y el criterio que se sigue para dividir zonas lingüísticas puede resultar subjetivo y con una fuerte impronta ideológica (Lara, 2007). 


\section{METODOLOGÍA}

A partir de una muestra representativa del alumnado de Bachillerato de Fuenlabrada, hemos repartido cuestionarios anónimos en cinco institutos públicos. Hemos reunido 338 cuestionarios pertenecientes a alumnos de $1 .^{\circ}$ y $2 .^{\circ}$ de Bachillerato que han rellenado en presencia del investigador. A continuación, se describe el perfil demolingüístico de la muestra y el procedimiento llevado a cabo en la extracción y análisis de los datos.

\subsection{Descripción de la muestra}

En esta investigación han participado 338 estudiantes de Bachillerato de Fuenlabrada, lo que supone una muestra representativa de este grupo poblacional con un nivel de confianza del $99 \%$ y un margen de error del 5 \% (Survey Monkey, 2018). Los rasgos demográficos de la muestra se detallan en la Tabla 1.

\begin{tabular}{|l|l|l|l|l|}
\hline \multirow{2}{*}{ Instituto } & \multicolumn{2}{c}{ Hombres } & \multicolumn{2}{c|}{ Mujeres } \\
& \multicolumn{2}{|c|}{$1 .{ }^{\circ} \mathrm{Bach}}$. & $2 .{ }^{\circ} \mathrm{Bach}$. & \multicolumn{2}{c|}{$2 .{ }^{\circ} \mathrm{Bach}}$. \\
IES Dolores Ibárruri & 0 & 23 & 0 & 41 \\
\hline IES Salvador Allende & 39 & 18 & 33 & 24 \\
\hline IES La Serna & 14 & 0 & 18 & 0 \\
\hline IES Carpe Diem & 18 & 0 & 17 & 0 \\
\hline IES Victoria Kent & 37 & 23 & 21 & 12 \\
\hline TOTAL (por curso y sexo) & $108(31,95 \%)$ & $64(18,93 \%)$ & $89(26,33 \%)$ & $77(22,79 \%)$ \\
\hline TOTAL (por sexo) & $172(50,88 \%)$ & \multicolumn{3}{|c|}{$166(49,12 \%)$} \\
\hline
\end{tabular}

Tabla 1. Cuantificación de la muestra representativa

No todos los institutos nos han ofrecido la oportunidad de acceder al alumnado de los dos cursos de Bachillerato. Sin embargo, dado que no centramos este estudio sobre la base de esta variable, solamente hemos procurado el mayor equilibrio posible. Contrariamente, el sexo de las personas participantes sí es relevante para los objetivos propuestos, por lo que hemos obtenido una proporción equidistante entre hombres y mujeres.

La singularidad de la procedencia extranjera no se revela como un dato sobresaliente puesto que esta población representa el 2,74\% de la muestra y alcanza unas cotas semejantes a las personas autóctonas respecto a la media de número de años 
vividos en Fuenlabrada - 15,18 años el alumnado extranjero y 15,25 el español一. La media de edad de la muestra de informantes es de 16,67 años, franja enmarcada etariamente en la adolescencia y subcatalogada como adolescencia tardía según organismos como UNICEF (2011). Durante esta etapa, el sujeto sustituye la presión normativa familiar y escolar por la influencia que ejerce el grupo de amigos u otros grupos sociales en los que se desenvuelve, lo cual implica la interacción con lenguas y formas lingüísticas no necesariamente prestigiosas (Chambers y Trudgill, 1980).

Respecto a la descripción lingüística de la muestra, el 91,42\% tiene como única lengua primera el español. Las lenguas de España distintas de esta se encuentran en 2 personas: una considera que el español y el catalán son sus lenguas maternas y otra declara que tan solo el rifeño lo es. El árabe, pese a considerarse una lengua autóctona del territorio español en tanto se encuentra en Ceuta, la hemos codificado como lengua extranjera puesto que las 4 personas que afirman tenerla como lengua materna han indicado Marruecos como lugar de nacimiento. Otras lenguas extranjeras con representación entre la muestra de informantes son el chino (9), el rumano (8) y el polaco (2). Por último, 3 personas afirman que tanto el español como otra lengua extranjera son sus lenguas maternas: búlgaro (2) y rumano (1).

\subsection{Procedimiento}

El acceso a la muestra de informantes ha sido posible gracias a la colaboración de las personas directivas de los institutos y de los responsables de diferentes departamentos que han coordinado las sesiones del trabajo de campo. La herramienta de extracción de datos ha consistido en cuestionarios anónimos rellenados en presencia del investigador tras explicar la temática y el objetivo del estudio. Estas aclaraciones se especificaban en el propio cuestionario, de tal modo que los discentes supieran en todo momento que no buscábamos interrogarles sobre sus conocimientos lingüísticos, sino más bien conocer sus impresiones y opiniones personales, desechando así la posibilidad de que entendiesen esta metodología como un examen con respuestas correctas e incorrectas.

Esta herramienta de trabajo se justifica atendiendo a varias razones: (i) permite recabar gran cantidad de datos de manera rápida y simultánea entre grupos de 2030 personas; (ii) permite dotar a las preguntas de un formato visual atractivo para los discentes en forma de tablas y escalas de valores, con enunciados breves y directos que evitan la apatía que les puede suscitar un trabajo académico; (iii) podemos obtener información a través de etiquetas precisas que denotan el posicionamiento respecto a la estética, la corrección o la adecuación de las variedades lingüísticas; y (iv) los datos son fácilmente codificables para su análisis posterior. 
En cuanto a la estructuración del cuestionario, un apartado introductorio recoge los datos demolingüísticos de la muestra y, posteriormente, se han diseñado dos bloques para conocer las ideologías sobre la diversidad lingüística de España y sobre la variación intradialectal del español. El primer bloque está destinado a cubrir una investigación paralela que trata el españolismo lingüístico a través de las ideologías referentes a la diversidad lingüística de España. Por lo tanto, para este trabajo solo hemos tenido en consideración el apartado introductorio y el segundo bloque (véase el ANEXo).

En este bloque, el cuestionario se compone de cuatro preguntas cerradas subdivididas en diferentes ítems. La primera actividad consiste en una escala de diferenciación semántica en la que la muestra ha de seleccionar en qué grado el español de Hispanoamérica y el de España suscitan características asociadas con sus hablantes, como la inteligencia, la simpatía o la sinceridad. En el segundo ejercicio, se enuncian preguntas directas sobre distintos prejuicios lingüísticos y se permite que los participantes respondan neutralmente escogiendo la opción «NS/NC». En tercer lugar, se propone una escala semántica para valorar la reacción afectiva que despiertan el acento estándar y un hipotético acento dialectal del español en torno a nociones de estética, corrección y adecuación. La cuarta actividad propone varios enunciados que representan ideologías lingüísticas muy marcadas y concisas para evaluar su aprobación o rechazo mediante una escala en la que el valor 1 implica estar totalmente en desacuerdo y el nivel 5 totalmente de acuerdo.

La codificación de los cuestionarios se ha realizado a través de la asignación de valores alfanuméricos a cada ítem y de números enteros a las respuestas. El tratamiento estadístico se ha llevado a cabo mediante el programa informático SPSS en el que se han operacionalizado los ítems y se han extraído porcentajes.

\section{ANÁLISIS DE LOS RESULTADOS}

\subsection{Juicios de lo hablado}

En la confrontación entre el español estándar y las variedades lingüísticas concretas, existe un paradigma sociocultural en la lingüistica hispánica que tiende al monolingüismo y a la supresión de variación idiomática como ideales a los que han de acercarse las comunidades humanas (del Valle y Gabriel-Stheeman, 2004). En consecuencia, el español estándar es presentado como la lengua legítima y correcta frente a la diversidad intradialectal y se le supone un carácter neutral y atemporal que sirve de modelo ante las demás variedades, las cuales, a su vez, son juzgadas bajo 
parámetros de estética, corrección y adecuación según se alejen o se aproximen al ideal del español estándar.

Con este panorama, investigamos las ideologías lingüísticas que presenta la muestra de informantes con respecto a los tres factores señalados anteriormente estética, corrección y adecuación- que se identifican en el conjunto de la variación intradialectal del español.

En primer lugar, algunas características como la sonoridad y la prosodia propias de cada variedad lingüística pueden despertar impresiones estéticas que las hacen ser juzgadas como más o menos bonitas o más o menos atractivas, por ejemplo. En el cuestionario se han propuesto el español con acento estándar y el español con acento dialectal para ser evaluados como hablas agradables o desagradables. Cabe señalar que el español con acento estándar alude a una variedad específica que los participantes pueden identificar en el español de Madrid, mientras que la etiqueta "español con acento dialectal" engloba infinidad de variedades difícilmente separables entre sí a partir de patrones fónicos. El acento estándar es agradable para el 73,67 \% de los encuestados y, aunque la proporción se reduce hasta el 53,55 \% en el caso del acento dialectal, no se percibe un rechazo reseñable a vincularlo con esta propiedad positiva. La circunscripción territorial de las variedades lingüísticas también es un factor que se toma en consideración en las valoraciones estéticas: el 63,31 \% de los informantes opina que el habla de las ciudades es más elegante que la de los pueblos.

En segundo lugar, la corrección idiomática es un factor que influye en la adopción de ideologías lingüísticas. A menudo, los hablantes se enfrentan ante la disyuntiva de asignar el rol de correcto a una sola forma gramatical, léxica o fonética cuando existen dos o más variantes lingüísticas. Milroy (2001) apunta que el criterio que se sigue se ve influenciado por la asunción de que, ante dos variantes, solo una puede ser correcta mientras que la otra es necesariamente incorrecta. En este sentido, es esperable que las personas informantes interpreten el acento estándar del español como el más correcto - el 65,68 \% lo corrobora-, pues la convención académica ha decidido que el estándar es el modelo ideal, aunque desde criterios estrictamente lingüísticos, no se puede medir la calidad o corrección de un hecho gramatical o fónico puesto que todos ellos cumplen una función comunicativa y son identificados por interlocutores que emplean las mismas variantes.

Como modelo paradigmático para ahondar en esta ideología, hemos propuesto el andaluz como variedad ante la que los participantes han de posicionarse. El $69,53 \%$ niega que las personas andaluzas hablen mal el español y el 58,28 \% no considera que esta variedad sea incorrecta por el hecho de «comerse las letras». Se rechaza así no solo un prejuicio que admite la falta de corrección en el habla andaluza, sino también el preconcepto del escriturismo por el cual se toma la lengua escrita 
como modelo al que ha de adaptarse la lengua oral (Moreno Cabrera, 2013). Este ejemplo arquetípico puede reforzarse si atendemos al 48,22\% de los participantes que considera que las palabras que no aparecen en el diccionario no son incorrectas, de modo que se percibe una distancia entre los usuarios de la lengua y los códigos normativos. Hay que tener en cuenta que la juventud en un sector lingüísticamente innovador y sus propuestas, a menudo importadas de lenguas como el inglés o formadas a través de otros recursos, no son aceptadas por la Academia hasta que su uso se hace extensivo a otros grupos etarios.

Estas relativas amplias proporciones de participantes que refutan dichas ideologías adquieren otra orientación cuando entran en juego los agentes institucionales que codifican la lengua. El 47,02\% identifica en la RAE una autoridad que vela por la calidad del español. Una posible explicación a esta aparente contradicción radicaría en lo que denominamos «reificación de la lengua».

La lengua, en este caso el español, sería vista como un objeto identificable en la externalidad de los hablantes y de las situaciones comunicativas. En tanto que objeto, estaría compuesto por una serie de elementos cuya relación entre sí permite el funcionamiento de todo el sistema. Esos elementos son las distintas categorías lingüísticas, como el léxico o los sonidos articulados, mientras que las reglas gramaticales ejercerían el rol de relación entre componentes que posibilita el funcionamiento del sistema lingüístico, es decir, de la lengua. Si volvemos a la metáfora de la lengua como una entidad reificada, los componentes de un objeto pueden romperse por un ensamblaje inadecuado e impedir su realización teleológica. Paralelamente, la ideología de la reificación de la lengua supone que la deturpación de las categorías lingüisticas, como por ejemplo una introducción incontrolada de vocablos foráneos, podría hacer tambalear todo el sistema y perder la esencia de la lengua. Como medida preventiva ante esta quiebra, existirían autoridades capaces de detectar a tiempo los peligros que amenazan la estabilidad del español y actuarían en consecuencia mediante una revisión de los componentes del sistema, como la omisión de ciertos préstamos lingüísticos en el diccionario, y la reestructuración de las relaciones entre los componentes, como la reorientación de las normas gramaticales.

Esta ideología no es incompatible con la negación del escriturismo dado que la especial atención de los usuarios a las reglas gramaticales puede basarse en los patrones orales y no escritos. Por su parte, la ideología de lo que estamos llamando «reificación de la lengua» tampoco parece entrar en contradicción con que la muestra de informantes considere que las palabras que no están recogidas en los códigos oficiales no son necesariamente incorrectas. La juventud puede emplear un léxico alternativo al que aparece en el diccionario de la RAE, y si esta institución niega la validez de dicho vocabulario, su legitimidad es cuestionada, pero no su- 
primida. Si asumimos la hipótesis de la reificación de la lengua, más allá de la propia RAE, la muestra de informantes no dispone de otro actor capaz de velar por el funcionamiento de la lengua, entendido como un sistema, y de activar mecanismos de regulación. Por ende, este grupo etario puede discrepar e incluso oponerse a determinadas decisiones de la Academia, pero su legitimidad permanece intacta dado que es percibida como un protector, tal y como demuestra una amplia proporción de informantes que consideran que la RAE vela por la calidad del español.

En tercer lugar, la variación intradialectal suscita connotaciones socioculturales de prestigio ante ciertas formas lingüísticas que llevan a tildar de inferiores o inadecuadas otras variantes subestándares (Hernández Campoy, 2004). Esta reputación es subjetiva y se atribuye por razones extralingüísticas como el origen geográfico o la clase social de los hablantes. De esta manera, las reacciones ideológicas que se despiertan favorecen o perjudican las racionalizaciones sobre las variedades lingüísticas y sobre sus hablantes.

La formalidad es una idea asociada a ciertas variedades que, en este estudio, adquiere unas cotas altas de neutralidad o desconocimiento cuando se valoran los acentos estándar y dialectal del español —en torno al $43 \%$ en ambos casos-. Sin embargo, quienes sí se posicionan lo hacen mayoritariamente a favor de conceder a ambos acentos la característica de formalidad, siendo más alto el consenso en torno al acento estándar - 55,33\% - que al dialectal - 32,25\%—. Por otro lado, cuando preguntamos si el español de los pueblos es adecuado en el ámbito formal, no podemos extraer ninguna tendencia clara, puesto que las tres respuestas que hemos dado a elegir representan, aproximadamente, un tercio de las opiniones de los informantes.

\subsection{Dialectalización del español}

Un prejuicio lingüístico habitual es el que sostiene que una variedad lingüística puede ser dialecto de un suprasistema con el que coexiste y al que se le asigna la categoría de «lengua». Cuando esta ideología sale a colación, cumple una función más sociopolítica que lingüística, pues sirve para enaltecer una de las variedades lingüísticas - normalmente la del poder político o económico-y desprestigiar otras, aludiendo a su supuesta carencia de cohesión y de capacidad expresiva. Desde un punto de vista diacrónico, podemos concluir que el español, el asturiano o el italiano, entre otros, son dialectos del latín, puesto que la evolución histórica del latín ha derivado en las lenguas mencionadas. Sin embargo, desde una perspectiva sincrónica, no se puede recurrir al término "dialecto" puesto que las hablas empleadas en un espacio y tiempo específicos no son ramificaciones las unas de las otras (Saussure, 1916). 
Habiendo considerado la funcionalidad meramente hegemónica cuando se alude a los dialectos como variedades imperfectas o coloquiales de las lenguas, la ciencia lingüística debe rebatir el argumento. Las lenguas son abstracciones de los elementos comunes de varias hablas, por lo que, en sentido estricto, sería más oportuno utilizar el concepto «dialecto», que es la variedad lingüística concreta y materializada en los eventos comunicativos, en lugar de «lengua» que, en tanto que abstracción, designa una realidad difícilmente identificable (Moreno Cabrera, 2013). La influencia cotidiana de diferentes entidades como los medios de comunicación provoca que la conciencia colectiva detente una impresión de los dialectos y las lenguas similar a una conceptualización diacrónica, aunque se estén tratando variedades lingüísticas en un momento histórico determinado.

En este sentido, se han realizado varias preguntas para averiguar el marco referencial de la muestra de informantes en torno a esta cuestión. En primer lugar, se ha propuesto el canario como variedad arquetípica que, previsiblemente, puede suscitar la reacción ideológica de catalogarlo como un dialecto. Tanto es así que cerca de la mitad de la muestra asegura que el canario es un dialecto del español - 47,63\%-, mientras que la otra mitad se reparte de modo parejo entre la negación de este supuesto - 27,22\%—y el desconocimiento - 25,15\%—.

En segundo lugar, este marco conceptual puede verse influido si la variedad lingüística que se considera no dispone de instituciones académicas que respalden su vitalidad. Hemos preguntado si las variedades sin gramática propia son dialectos. En la propia formulación del enunciado se halla una premisa falsa, pues la gramática es el conjunto de patrones a partir de los que se forman palabras y estas se combinan entre sí para construir mensajes, independientemente del hipotético amparo de un organismo académico que haya elaborado una gramática física de carácter descriptivo o prescriptivo. El grado numérico más repetido de los que se han propuesto para indicar conformidad o disconformidad ha sido el que marca neutralidad - 54,44\%— que, por ser el más imparcial, también puede denotar desconocimiento ante una cuestión sobre la que las personas participantes no se hayan detenido a reflexionar previamente. Por su parte, el 26,63\% afirma que las hablas sin gramática son dialectos y el $18,93 \%$ niega este prejuicio.

En tercer lugar, cuando indagamos en las ideologías respecto al hipotético descuido y dejadez de las personas en su habla coloquial, la mitad de la muestra no se involucra - 49,11\%—, pero un destacable 33,14\% apunta que se trata de una desviación de la lengua estándar. Esta porción de la muestra encuentra en el habla coloquial elementos degradados de la lengua estándar, por lo que interpreta esta última como ideal de corrección y aquella como una versión deturpada por la desidia de los hablantes. 
Por último, hemos aprovechado una muestra de informantes eminentemente de procedencia madrileña para cuestionar la ideología del español neutro. Este término surgió en la década de 1960 para aludir a una variedad carente de regionalismos y de trazos lingüísticos que se pudiesen asociar con variedades concretas. Su tratamiento no ha tenido una amplia repercusión en la sociolingüística dado su eminente motivación comercial de expansión empresarial (Castro Roig, 1996). Este concepto ha sido empleado en la industria de la traducción y del doblaje de productos audiovisuales, transmitiéndose así a la conciencia colectiva de la población como un español regular y sin marcas dialectales (Iparraguirre, 2014). Algunos autores han detectado el origen de este preconcepto en los medios de comunicación que, ante la necesidad de homogeneizar la lengua y suprimir o minimizar las marcas diatópicas y diastráticas recurren a un pretendido lenguaje neutral que garantizaría la comprensibilidad a los hablantes de todas las variedades (Petrella, 2006). La identificación de un español exento de rasgos idiosincráticos puede acarrear la minusvaloración de las demás variedades de la lengua que, al fin y al cabo, son las hablas naturales de cada comunidad lingüística. En este sentido, la opinión mayoritaria de nuestra muestra de informantes confirma la tesis de que las personas madrileñas tienen un acento neutro - 47,34\%-, mientras que el $36,98 \%$ no se involucra en esta problemática y apenas un $15,68 \%$ la rechaza.

\subsection{Valoraciones sobre los hablantes}

Mediante una escala semántica, hemos pedido a las personas participantes que indicasen en qué medida están de acuerdo con cuatro pares de adjetivos asociados a los hablantes de español de España y de Hispanoamérica. De esta manera, siguiendo el modelo de Haddock (2004) respecto a la medición de los juicios evaluativos, podemos indagar tanto en la valencia que adquieren las ideologías como en la intensidad de las mismas en torno a la inteligencia, la simpatía, la sinceridad y la fiabilidad.

Respecto a las personas hispanoamericanas, la simpatía es la característica positiva que se les concede de manera más clara $-57,4 \%$ - ya que el porcentaje de las demás propiedades asociadas con valores positivos se mueve entre el $20 \%$ y el $30 \%$. Estas proporciones no implican ideologías generalizadas de aversión y desprestigio hacia los hablantes hispanoamericanos, puesto que las cotas más altas se dan en la posición intermedia. Con una media de 54,81\% entre los cuatro atributos presentados, se observa la predisposición de la muestra de informantes a mantenerse al margen de concesiones psicológicas. Por consiguiente, a la hora de juzgar a los hablantes hispanoamericanos, la inclinación detectada es aceptar que la variedad de español no conlleva aptitudes psicológicas específicas, puesto que la lengua es un fenómeno independiente de la personalidad. 
Sin embargo, esta ligera mayoría en la escala intermedia decrece cuando se trata de evaluar a los hablantes de español de España, ideología que alcanza el 49,34 \% de los encuestados. En este caso, observamos disposiciones más militantes, generalmente para conceder a estos hablantes cualidades positivas. A pesar de ello, en ningún caso estos hablantes son vinculados con dichas propiedades con proporciones superiores al $60 \%$, lo cual confirma y completa la hipótesis mencionada anteriormente: la personalidad de un colectivo de hablantes es interpretada como una variable externa y no dependiente de la lengua propia de dicha comunidad, pero si la persona evaluadora forma parte de la comunidad lingüística que está juzgando, ambos factores - la lengua y la personalidad - pueden acercarse y las representaciones que genera tienden a adquirir autopercepciones más positivas que cuando evalúa una comunidad externa.

\section{CONCLUSIONES}

En esta investigación, hemos ahondado en la concepción que tiene una muestra representativa del alumnado de Bachillerato de Fuenlabrada respecto a la variación lingüística que presenta el español habida cuenta de su extensión transnacional.

De entre las características potencialmente asociables a las variedades lingüísticas concretas, la propiedad estética es la que mayor consenso despierta. La cualidad de «agradable», configurada a partir de impresiones subjetivas, es asumida como genuina del acento estándar y urbano. Paralelamente, el español estándar representa el ideal de corrección, aunque no por ello parece existir un desprestigio de otras variedades lingüísticas. En este sentido, hemos propuesto la hipótesis de la «reificación de la lengua», entendida como una ideología que asume la lengua como un objeto externo a los hablantes y manipulable a través del dictamen de los doctos organismos académicos. A pesar de que el vocabulario y las formas lingüísticas propias de la juventud puedan contrastar con los códigos oficiales impulsados por la RAE, esta institución parece gozar de una reputación tal que la lleva a ser percibida como la salvaguarda del español.

Por otro lado, se percibe el preconcepto que admite la existencia de dialectos, interpretados como ramificaciones coetáneas a una pretendida lengua general. La oposición entre estos conceptos es asimétrica, puesto que la lengua se vincula con un ideal al que han de acercarse los hablantes, mientras que los dialectos estarían motivados por el descuido de los usuarios. Esta ideología se relaciona con el prescriptivismo característico de las academias de la lengua que, en lugar de describir los fenómenos lingüísticos que acontecen en la cotidianeidad, generan códigos del buen hablar, 
de manera que aquellas formas lingüísticas alejadas de ese estándar elaborado son percibidas por la comunidad como degradaciones. De entre las múltiples variedades del español, se constata la predilección por el madrileño como acento neutro, como una idea de español general que traspasa fronteras.

Por último, se comprueba la disociación entre la variedad del español y las características psicológicas de los hablantes, por lo que ambos factores son entendidos como independientes. No obstante, hemos detectado una mayor tendencia a acercar estas dos variables cuando se trata de una autorrepresentación como grupo diferenciado. Las opiniones sobre la comunidad lingüística en la que se identifican las personas participantes adquieren un cariz más militante y, dentro de este, mayoritariamente positivo, ante las propiedades psicológicas propuestas.

A partir de los datos expuestos, parece confirmarse la vigencia de la ideología hegemónica del panhispanismo. En las últimas décadas, instituciones como la RAE han tratado de desvincularse de una tradición anterior asociada al prescriptivismo y a la óptica de modelo lingüístico desde la centralidad en España. Sin embargo, esas ideas perviven en las racionalizaciones lingüísticas de un grupo poblacional que, por su condición etaria, podría estar más predispuesta al reconocimiento de otras formas lingüísticas.

\section{BIBLIOGRAFÍA}

AlineI, Mario y Wolfgang Viereck (1997): Atlas Linguarum Europae: perspectives nouvelles en géolinguistique, Roma, Istituto Poligrafico e Zecca dello Stato.

Amorós Negre, Carla (2008): Norma y estandarización, Salamanca, Luso-Española de Ediciones.

BERNÁRDEZ, Enrique (2010): «Spanish: A centreless multicentred language?», International Conference on Pluricentric Languages: Linguistic Variation and Sociocognitive Dimensions, Braga, Catholic University.

Blanco, Ana (2004): Estudio sociolingüistico de Alcalá de Henares, Alcalá de Henares, Universidad de Alcalá.

Castro Roig, Xosé (1996): «El español neutro», en Asociación Estadounidense de Traductores (org.), Congreso anual de la ATA, Colorado Springs.

Cestero, Ana María; Isabel Molina y Florentino Paredes (2006): Estudios sociolingüisticos del español de España y América, Madrid, Arco/Libros.

Chambers, Jack y Peter Trudgill (1980): Dialectology, Cambridge, Cambridge University Press. 
Contini, Michel y Gaston Tuaillon (1996-2009): Atlas Linguistique Roman (ALiR), Roma, Istituto Poligrafico e Zecca dello Stato.

Del VAlle, José (2007a): «La lengua, patria común: la hispanofonía y el nacionalismo panhispánico», en José del Valle (ed.), La lengua, ¿patria común? Ideas e ideologías del español, Madrid/Frankfurt, Iberoamericana/Vervuert, pp. 31-56.

Del VAlle, José (2007b): La lengua, ¿patria común? Ideas e ideologías del español, Madrid/Frankfurt, Iberoamericana/Vervuert.

Del Valle, José y Luis Gabriel-Stheeman (2004): «Nacionalismo, hispanismo y cultura monoglósica », en José del Valle y Luis Gabriel-Stheeman (eds.), La batalla del idioma. La intelectualidad hispánica ante la lengua, Madrid/Frankfurt, Iberoamericana/Vervuert, pp. 15-33.

García Mouton, Pilar (2011): «Actitudes lingüísticas de mujeres - y de hombres- en el Madrid rural», en Lidia Taillefer de Haya (ed.), La igualdad: nuevas perspectivas de género en educación, lingüistica y filosofía, Málaga, CEDMA, pp. 183-208.

García Mouton, Pilar (2007): «Vitalidad y mortandad léxica en las hablas rurales de Madrid», en Josefa Dorta (ed.), Temas de dialectología, La Laguna, Instituto de Estudios Canarios, pp. 81-93.

García Mouton, Pilar y Isabel Molina Martos (2009): «Trabajos sociodialectales en la Comunidad de Madrid», Revista de Filología Española, 89, 1, pp. 175-186.

Gil PeÑA, Noelia (2004): Estudio sociolingüistico de la fonética de la ciudad de Madrid, Tesis de licenciatura, Madrid. Universidad de Alcalá.

Haddock, Geoffrey (2004): «On Using Questionnaires to Measure Attitudes», en Glynis M. Breakwell (ed.), Doing Social Psychology Research, Malden, BPS Blackwell, pp. 154-173.

Hernández Campoy, Juan Manuel (2004): «El fenómeno de las actitudes y su medición en sociolingüística », Tonos digital. Revista electronica de estudios filológicos, 8. En línea: <https://dialnet.unirioja.es/servlet/articulo?codigo $=1049699>[25 / 11 / 2018]$.

IpARRAGUiRre, Carolina (2014): «Hacia una definición del español neutro», Síntesis, 5, pp. 232-252.

IRVINE, Judith (1989): «When talk isn't cheap: language and political economy», American Ethnologist, 16, 2, pp. 248-267.

Kroskrity, Paul V. (2010): «Language ideologies - Evolving Perspectives», en Jürgen Jaspers, Jan-Ola Östman y Jef Verschueren (eds.), Society and Language Use, Ámsterdam/Filadelfia, John Benjamins Publishing Company, pp. 192-211. 
LARA, Luis F. (2007): «Por una reconstrucción de la idea de la lengua española. Más allá de las fronteras instituidas», en José del Valle (ed.), La lengua, ¿patria común? Ideas e ideologías del español, Madrid/Frankfurt, Iberoamericana/Vervuert, pp. 163-181.

LAUria, Daniela y María López García (2009): «Instrumentos lingüísticos académicos y norma estándar del español: la nueva política lingüística panhispánica », Lexis, 33, 1, pp. 49-89.

Martín Butragueño, Pedro (2001): «El contacto de dialectos como motor del cambio lingüístico», en Instituto Cervantes (ed.), Actas del II Congreso Internacional de la Lengua Española, Valladolid, Instituto Cervantes. En línea: <http://congresosdelalengua.es/valladolid/> [02/01/2019].

Martín Butragueño, Pedro (1993): «Actitudes y creencias lingüísticas en inmigrantes dialectales: el caso de Madrid»,Lingüistica Española Actual, 15, 2, pp. 265-296.

Mertz, Elizabeth (2010), «Linguistic Ideology and Praxis in U.S. Law School Classrooms», en Bambi B. Schieffelin; Kathryn A. Woolard y Paul V. Kroskrity (eds.), Language Ideologies. Practice and Theory, Oxford, Oxford University Press, pp. 149-162.

Milroy, James (2001): «Language ideologies and the consequences of standardization », Journal of Sociolinguistics, 5, 4, pp. 530-555.

Molina, Isabel (2009): «Sociolingüística del español en Madrid», Liceus. En línea: <https://aprende.liceus.com/producto/sociolinguistica-del-espanol-ma$\mathrm{drid} />[20 / 12 / 2018]$.

Molina, Isabel (2006): «Innovación y difusión del cambio lingüístico en Madrid», Revista de Filología Española, 86, 1, pp. 175-186.

Molina, Isabel (2005): «Estrategias de atenuación en el barrio de Salamanca de Madrid», en ALFAL (ed.), XIV Congreso Internacional ALFAL. Memorias, Monterrey, Universidad Autónoma de Nuevo León, vol. I, pp. 212-220.

Moreno Cabrera, Juan Carlos (2013): Cuestiones clave de la lingüistica, Madrid, Síntesis.

Moreno Cabrera, Juan Carlos (2011): «"Unifica, limpia y fija.” La RAE y los mitos del nacionalismo lingüístico español», en Silvia Senz y Montserrat Alberte (eds.), El dardo en la Academia. Esencia y vigencia de las academias de la lengua española, Barcelona, Melusina, vol. I, pp. 157-314.

PAREDES, Florentino (2006): «Dialectología y sociolingüística en Madrid», en Ana María Cestero, Isabel Molina y Florentino Paredes (eds.), Estudios sociolingüisticos del español de España y América, Madrid, Arco/Libros, pp. 221231. 
PAREDES, Florentino (2005): «El léxico de los colores: convergencias y divergencias en grupos sociales de Madrid», en ALFAL (ed.), XIV Congreso Internacional ALFAL. Memorias, Monterrey, Universidad Autónoma de Nuevo León, vol. I, pp. 131-140.

Penny, Ralph (2000): Variación y cambio en español, Madrid, Gredos.

Peralta Céspedes, Manuel (2017): Convergencia y divergencia en el español de hablantes dominicanos en Madrid, Tesis de doctorado, Madrid, Universidad Complutense de Madrid.

Petrella, Lila (2006): «El controvertido concepto de español neutro. Reflexiones desde el léxico», en IV Congreso Internacional "Transformaciones culturales. Debates de la teoría, la critica y la lingüistica”, Buenos Aires, Facultad de Filosofía y Letras.

Quilis, Antonio (1966): «Notas para el estudio del habla de Madrid y su provincia $\gg$, Anales del Instituto de Estudios Madrileños, I, pp. 365-372.

SANCho PAscual, María (2015): «Integración sociolingüística de los inmigrantes ecuatorianos en Madrid: datos sobre el diminutivo según los corpus Ispie-Madrid y PRESEEA-Madrid», en Ana María Cestero, Isabel Molina y Florentino Paredes (eds.), Patrones sociolingüisticos de Madrid, Berna, Peter Lang, pp. 155176.

SAussure, Ferdinand de (1916): Cours de linguistique générale, París, Payot.

Silverstein, Michael (1979), «Language structure and linguistic ideology», en Paul R. Clyne, William F. Hanks y Carol L. Hofbauer (eds.), The Elements: A Parasession on Linguistic Units and Levels, Chicago, Chicago Linguistic Society, pp. 193-247.

Solís CAsco, Isabel María (2015): Aulas de enlace: un análisis de las politicas lingüisticas educativas en la Comunidad de Madrid, Tesis de doctorado, Madrid, UNED.

Survey Monkey (2018). En línea: <http://www.surveymonkey.com> [08/11/2018].

Torres Martínez, José Carlos de (1981): Encuestas léxicas del habla culta de Madrid, Madrid, CSIC.

UNICEF (2011): Estado mundial de la infancia 2011. La adolescencia: una época de oportunidades, Nueva York, UNICEF. 


\section{ANEXo}

\section{CUESTIONARIO SOBRE IDEOLOGÍAS LINGÜÍSTICAS}

Este cuestionario trata sobre las percepciones y opiniones personales que tiene el alumnado de Bachillerato de Fuenlabrada con respecto a la diversidad lingüística de España y a las variedades del español. No hay respuestas correctas ni incorrectas, por lo que, simplemente, te pedimos que respondas con la mayor sinceridad posible. Se trata de un cuestionario anónimo y los datos que extraigamos serán utilizados con fines exclusivamente académicos y estadísticos. Por último, queremos agradecer tu participación, sin la cual este proyecto no sería posible.

\section{INTRODUCCIÓN}

1. Sexo:

2. Edad:

3. Lugar de nacimiento:

$\square$ Andalucía $\square$ Aragón $\square$ Asturias $\square$ Baleares $\square$ Canarias $\square$ Cantabria $\square$ Castilla-La Mancha $\square$ Castilla y León $\square$ Cataluña $\square$ Ceuta $\square$ Extremadura $\square$ Galicia $\square$ La Rioja $\square$ Madrid $\square$ Melilla $\square$ Murcia $\square$ Navarra $\square$ País Vasco $\square$ Valencia

$\square$ Otro país (indica cuál):

4. ¿Cuántos años llevas viviendo en Fuenlabrada?

5. ¿Cuál es tu lengua materna?

\section{BLOQUE 2: VARIEDADES DEL ESPAÑOL}

11. Marca con una $\mathrm{x}$ las características que asignarías a los hablantes de las siguientes variedades de español:

\begin{tabular}{|l|l|l|l|l|l|}
\hline \multicolumn{3}{|c|}{ HABLANTES DE ESPAÑOL DE LATINOAMÉRICA } \\
\hline Muy poco inteligentes & & & & & Muy inteligentes \\
\hline Muy poco simpáticos & & & & & Muy simpáticos \\
\hline Muy poco sinceros & & & & & Muy sinceros \\
\hline Muy poco fiables & & & & & Muy fiables \\
\hline
\end{tabular}




\begin{tabular}{|l|l|l|l|l|l|}
\hline \multicolumn{7}{|c|}{ HABLANTES DE ESPAÑOL DE ESPAÑA } \\
\hline Muy poco inteligentes & & & & & Muy inteligentes \\
\hline Muy poco simpáticos & & & & & Muy simpáticos \\
\hline Muy poco sinceros & & & & & Muy sinceros \\
\hline Muy poco fiables & & & & & Muy fiables \\
\hline
\end{tabular}

12. Selecciona la respuesta que más se ajuste a tus creencias ("sí", "no", "no sabe / no contesta"):

- ¿Consideras que el canario es un dialecto del español?

$\square$ Sí $\square$ No $\square$ NS/NC

- ¿Consideras que los andaluces hablan mal el español?

$\square$ Sí $\square$ No $\square$ NS/NC

- ¿Los madrileños tienen un acento neutro?

$\square$ Sí $\quad \square$ No $\square$ NS/NC

- ¿Las hablas de las ciudades son más elegantes que las hablas de los pueblos? $\square$ Sí $\square$ No $\square$ NS/NC

- ¿El español de España es de mejor calidad que el español de Latinoamérica? $\square$ Sí $\square$ No $\square$ NS/NC

13. Marca con una $\mathrm{x}$ las características que asignarías a las siguientes variedades de español:

\begin{tabular}{|l|l|l|l|l|l|l|}
\hline \multicolumn{7}{|c|}{ ESPAÑOL CON ACENTO ESTÁNDAR } \\
\hline Desagradable & & & & & & Agradable \\
\hline Informal & & & & & & Formal \\
\hline Incorrecto & & & & & & Correcto \\
\hline
\end{tabular}

\begin{tabular}{|l|l|l|l|l|l|l|}
\hline \multicolumn{7}{|c|}{ ESPAÑOL CON ACENTO DIALECTAL } \\
\hline Desagradable & & & & & & Agradable \\
\hline Informal & & & & & & Formal \\
\hline Incorrecto & & & & & & Correcto \\
\hline
\end{tabular}

14. Puntúa las siguientes afirmaciones del 1 al 5 según este esquema:

$1=$ totalmente en desacuerdo, 2 = más bien en desacuerdo, $3=$ ni de acuerdo ni en desacuerdo, $4=$ más bien de acuerdo, 5 = totalmente de acuerdo 
- El andaluz no es correcto porque los andaluces se comen letras al hablar.

- Los acentos del español de los pueblos no son adecuados en ámbitos formales.

- Las palabras que no están en el diccionario son incorrectas.

- La Real Academia Española vela por la calidad del español.

- El habla coloquial es una desviación del español estándar.

- Las hablas que no tienen gramática propia son dialectos.

Fecha de recepción: 1 de mayo de 2019 Fecha de aceptación: 2 de octubre de 2019 